\title{
Generation of Ultra-Short Microwave Pulses in a Tunable Gyrotron with Subsequent Compression
}

\author{
$\underline{\text { R.M. Rozental }}^{1}$, I.V. Zotova ${ }^{1}$, N.S. Ginzburg ${ }^{1}$, V.P. Tarakanov ${ }^{2,3}$ \\ ${ }^{1}$ Institute of Applied Physics RAS, Nizhny Novgorod, Russia, rrz@appl.sci-nnov.ru \\ 2 Moscow Engineering Physics Institute, Moscow, Russia \\ ${ }^{3}$ Joint Institute for High Temperatures, RAS, Moscow, Russia
}

Generation of ultrashort electromagnetic pulses is of interest for many applications. A well-known method for obtaining such pulses is based on the compression of a frequency-modulated signal in a dispersive medium [1]. In principle, as such media, one can use regular waveguides. However, in this the frequency range optimal for compression is very close to the critical frequency, where the dispersion of the wave is most pronounced. This creates problems for ensuring matching in the operating band of the input chirped source. In [2,3] for solution of this problem, the compression in the helical waveguides is proposed. But in high-frequencies band such systems are rather complicated for manufacturing.

In this paper we suggest the two-stage scheme of generation of multi kilowatts subnanosecond microwave pulses based on a gyrotron matching directly with a compression section in the form of a regular waveguide. It is well-known, that gyrotrons typically have a rather narrow band of frequency tuning ( $\sim$ 0.1$0.3 \%$ ). However, as it was shown in [4], significant widening of the generation band in gyrotrons can be achieved with using of a shortened interaction space due to the weaker sensitivity to the velocity spread in the electron beam. The required frequency tuning of $5 \%$ can be provided due to excitation of several axial modes.

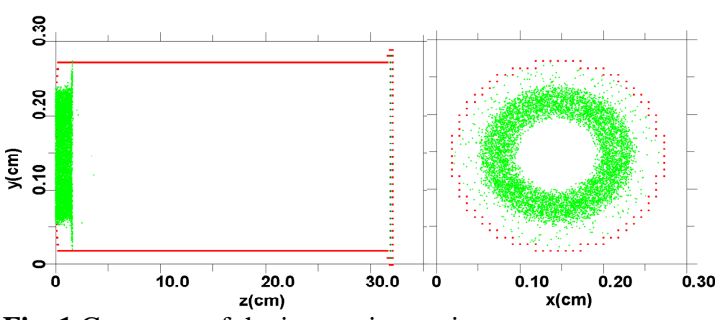

Fig. 1 Geometry of the interaction region.

Further, results of particle-in-cell simulations of such a system are presented based on the KARAT code [5]. In Fig.1 the geometry of the interaction region is shown, where the first section with an electron beam corresponds to a gyrotron with rather short resonator, while the second one is the section of compression. The Ohmoc losses were taken into account. The electron beam with the current of $2 \mathrm{~A}$, excites the $\mathrm{TE}_{21}$ mode at the frequency of about $200 \mathrm{GHz}$. the operating Due to variation of an accelerating voltage from 90 to $15 \mathrm{kV}$, the operating frequency of a gyrotron varies from 200 to $210 \mathrm{GHz}$ in the pulse with a duration of $12 \mathrm{~ns}$ (Fig.2a). The radiation power at the output of a gyrotron doesn't exceed $8 \mathrm{~kW}$. In the second stage, the radiation is compressed in a regular waveguide connected directly to the gyrotron resonator. As a result, the output radiation represents the subnanosecond pulse with power of $80 \mathrm{~kW}$.

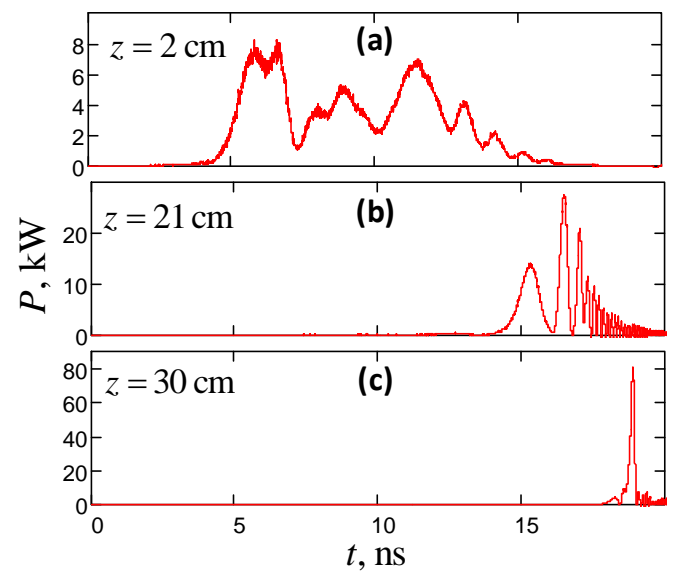

Fig. 2. Evolution of the radiated pulse in the section of compression.

This research was performed within the framework of the RFBR project No. 18-08-00717.

\section{References}

1. Petelin, M. Microwave pulse compressors // Proc. Int. Workshop on Strong Microwaves in Plasmas. 1997. V. 2. P. 903.

2. Samsonov, S.V., Phelps, A.D.R., Bratman, V.L., Burt, G., Denisov, G.G., Cross, A.W., Ronald, K., He, W., Yin, H. Compression of Frequency-Modulated Pulses using Helically Corrugated Waveguides and Its Potential for Generating Multigigawatt rf Radiation // Phys. Rev. Lett. 2004. V. 92. No.11. Art.no. 118301.

3. Bratman, V.L., Denisov, G.G., Kolganov, N.G., Mishakin, S.V., Samsonov, S.V., Cross, A.W., He, W., Zhang, L., McStravick, M.C., Whyte, G., Young, A.R., Ronald, K., Robertson, C.W., Phelps, A.D.R. Generation of $3 \mathrm{GW}$ microwave pulses in X-band from a combination of a relativistic backward-wave oscillator and a helicalwaveguide compressor // Phys. Plasmas. 2010. V. 17. No. 11. Art.no. 110703.

4. Fedotov, A.E., Rozental, R.M., Zotova, I.V., Ginzburg, N.S., Sergeev, A.S., Tarakanov, V.P., Glyavin, $M . Y u$., Idehara T. Frequency Tunable sub-THz Gyrotron for Direct Measurements of Positronium Hyperfine Structure // J Infrared Milli Terahz Waves. 2018. V. 39. No. 10. P. 975.

5. Tarakanov, V.P. Code KARAT in simulations of power microwave sources including Cherenkov plasma devices, vircators, orotron, E-field sensor, calorimeter etc. // EPJ Web of Conferences. 2017. V. 331. Art.no. 04024. 